\title{
"What” Becoming "Where": Functional Magnetic Resonance Imaging Evidence for Pragmatic Relevance Driving Premotor Cortex
}

\author{
Uta Wolfensteller, Ricarda I. Schubotz, and D. Yves von Cramon \\ Department of Cognitive Neurology, Max Planck Institute for Human Cognitive and Brain Sciences, 04303 Leipzig, Germany
}

\begin{abstract}
Previous studies using the serial prediction task (SPT) have shown that attending to the locations of objects activates the dorsal part of premotor cortex more than attending to the sizes of objects. The opposite holds for the ventral part of the premotor cortex. The present study used functional magnetic resonance imaging to investigate whether the learning of arbitrary stimulus-response mappings influences this functional dissociation. One experimental group learned to assign stimuli to response buttons based on stimulus size; another group did so based on stimulus location. More specifically, one-half of the participants in both experimental groups learned to assign stimuli to finger movements of their right hand, whereas the other half assigned stimuli to finger movements of their left hand. During scanning, all participants performed both size SPT and location SPT. Thus, we investigated the effects of the attended stimulus property (size or location), the motor effector assigned to it (fingers of left or right hand), and the spatial arrangement of the targets (the same in all groups). As expected, without motor training, the dorsal premotor cortex was less activated during size SPT compared with location SPT. The opposite held for ventral premotor cortex. With motor training, however, this differential activity pattern vanished. Activity in dorsal premotor cortex reflected neither the attended stimulus property nor the motor effector assigned to it. Instead, its activity may be related to the spatial properties of the response targets once some object property, such as size, takes on the "pragmatic relevance" of a spatially directed response.
\end{abstract}

Key words: fMRI; nonstandard mapping; object attention; premotor cortex; sensorimotor; sequence; spatial attention

\section{Introduction}

The lateral premotor cortex (PM) is involved in processes that often precede an action, including attentional tuning and sensorimotor transformation. Monkey studies suggest that the superior ventral and dorsal portions of PM (area F4 and area F2, respectively) are selectively involved in the processing of spatial targets and the planning of reaching movements, whereas the inferior ventral PM (area F5) is rather tuned to object properties and the planning of grasping and manipulation movements (Luppino et al., 1999; Lebedev and Wise, 2001; Rizzolatti and Luppino, 2001). Interestingly, functional magnetic resonance imaging (fMRI) studies imply a similar correspondence in humans, linking more dorsal PM to spatial properties and more ventral PM to objects, even in purely attentional tasks (Schubotz and von Cramon, 2001, 2002a,b,c; Schubotz et al., 2003). These findings suggest that attention to spatial properties activates the

Received July 2, 2004; revised Sept. 28, 2004; accepted Oct. 4, 2004.

We thank Shirley-Ann Rueschemeyer and Jan-Peter Herrmann for proofreading and suggestions; Birte Forstmann, Thomas Jacobsen, and Katrin Sakreida for helpful comments on this manuscript; Gabi Lohmann and Karsten Müller for support in MRI statistics; Bettina Johst for support in programming; and Ramona Menger, Anke Mempel, and Mandy Naumann for experimental assistance.

Correspondence should be addressed to Uta Wolfensteller, Department of Cognitive Neurology, Max Planck Institute for Human Cognitive and Brain Sciences, P.0. Box 500 355, 04303 Leipzig, Germany. E-mail: wolfen@cbs.mpg.de.

D0I:10.1523/JNEUROSCI.2641-04.2004

Copyright $\odot 2004$ Society for Neuroscience $\quad 0270-6474 / 04 / 2410431-09 \$ 15.00 / 0$ premotor areas for reaching and gaze, whereas attention to objects activates the premotor areas for grasping.

However, PM is also known to be engaged in so-called nonstandard mappings, or the mapping of an arbitrary stimulus onto an arbitrary movement (Mitz et al., 1991; Wise et al., 1996; Murray et al., 2000). Nonstandard mappings allow for great behavioral flexibility in everyday life: applying pressure with the right foot to one of several pedals in response to a red but not a green light is an example of a rather arbitrary coupling, but when seen in the context of traffic safety, it takes on vital importance. Acquiring a nonstandard mapping generally requires the integration of information from three factors: (1) properties of the tobe-attended stimulus (e.g., the redness of the traffic light), (2) the motor effector (the right foot), and (3) the target property (the brake pedal).

Given the preference of dorsal PM for attention to object locations (vs sizes), the preference of ventral PM for attention to object sizes (vs locations), and the hypothesized role of PM in arbitrary mappings, we wanted to know whether the learning of arbitrary mappings would change the attentional preferences. The present study used fMRI to investigate whether the PM correlates of size and location attention are affected by the motor effector or by the target property of a nonstandard mapping. In a pre-experimental training protocol, one-half of the participants were trained to push one of four horizontally arranged buttons, depending on the size of the presented stimuli. One-half of the 
participants in this group gave responses with their left hand, and one-half gave responses with their right. The other participants saw the same stimuli but learned to push one of four horizontally arranged buttons, depending on the location of the presented stimuli. Again, one-half of the participants in this group responded with the left hand and one-half with the right hand. Hence, participants in the four groups acquired different arbitrary mappings between the to-be-attended stimulus property (size, location), the motor effector (right hand, left hand), and the target property (same spatial button arrangement for all groups). In the subsequent fMRI session, all participants performed a location attention task and a size attention task, which are known to engage the PM [serial prediction task paradigm (SPT)] (Schubotz, 1999).

According to previous findings (Schubotz and von Cramon, 2003; Schubotz et al., 2003), we expected that attending to size would elicit activation in the ventral PM, whereas attending to location would elicit activation in the dorsal PM (hypothesis 1). Provided that this typical activation pattern would change when participants attend to a stimulus property, for which they had acquired a nonstandard mapping beforehand, two patterns could be expected in principle. If the motor effector used in training (i.e., the hand) is reflected in PM, then premotor correlates should differ between participants trained with the right hand and those trained with the left hand. Specifically, we should see the contralateral PM to be dominantly engaged (hypothesis 2). If, in contrast, PM activity reflects the spatial arrangement of the response buttons, then activity there should no longer differ between participants with size training and those with location training. Instead, following the idea that spatial attention and sensorimotor transformation engage rather dorsal premotor areas (Hoshi and Tanji, 2004), dorsal PM should be activated in both groups as a result of the spatial response mapping (hypothesis 3).

\section{Materials and Methods \\ Participants}

Thirty-two participants (17 females; mean age, 24.6; range, 21-28) took part in this experiment. All participants gave written consent before being included in testing. All participants were right handed, had normal or corrected-to-normal vision, and were native German speakers. No participant had a history of neurological, psychiatric, or other major medical disorder. No participant was taking medication at the time of measurement. The experimental standards were approved by the local ethics committee of the University of Leipzig (Leipzig, Germany). Data were handled anonymously.

\section{Design}

Participants were pseudorandomly assigned to the cells of a $2 \times 2$ between-subjects design (compare Fig. 1, Fig. 2, gray cells) with the factors training (size, location) and motor effector (left, right). Training refers to the stimulus property for which participants learned a sensorimotor mapping (detailed below). Motor effector refers to the hand with which participants were trained and thus the laterality of the learned sensorimotor mapping. Participants were assigned to each group in such a manner that gender was balanced across experimental groups. Participants learned a sensorimotor mapping for only one of the stimulus properties (either size or location). However, in the experiment, both stimulus properties had to be attended to in different trials (see Trials). Therefore, the two-level factor attended property (size, location) was introduced within participants (Fig. 2, columns in design table) to distinguish between attending to stimuli for which a sensorimotor mapping had been trained and attending to stimuli for which no sensorimotor mapping had been trained. The resulting $2 \times 2 \times 2$ mixed factorial design is depicted in Figure 2.
Non-standard mapping

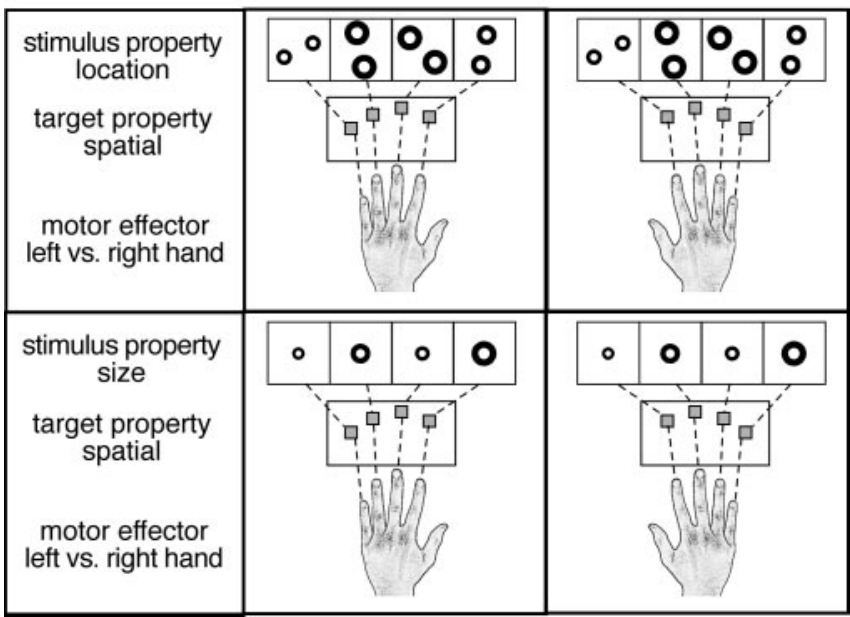

Figure 1. Nonstandard mapping. Four experimental groups were trained to associate either locations (top) or sizes of objects (bottom) to button presses with either the left (left) or the right hand (right). Note that the target property was spatial for all groups, because the buttons were horizontally arranged. Stimulus-response compatibilities were balanced by using four different mappings for each stimulus property. The figure depicts only one of each as an example. Stimuli in both the size-response mapping and the location-response mapping comprised two identical circular forms. To enhance discriminability of sizes, only one circular form is depicted for the size-response mapping.

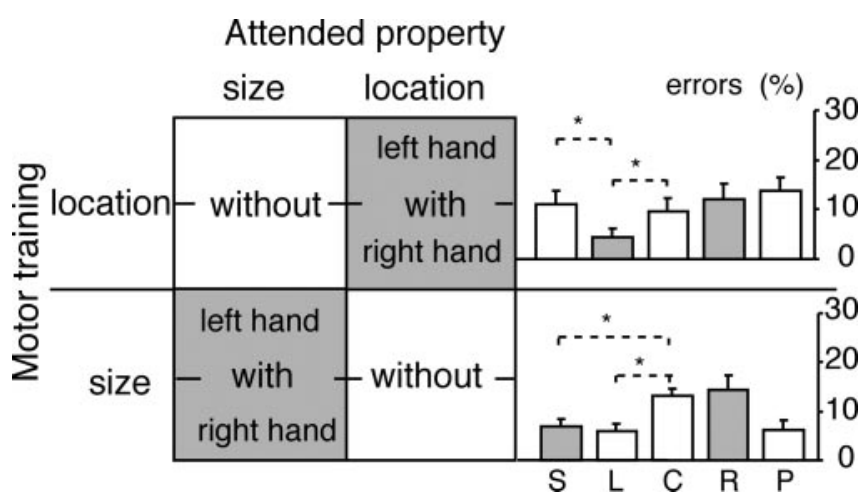

Figure 2. Experimental design and behavioral performance. The 2 (trained stimulus property) $\times 2$ (trained hand) $\times 2$ (attended stimulus property) mixed factorial design is depicted on the left. Behavioral task performance is depicted separately for participants with a motor training for locations (top) and sizes (bottom) on the right. Bars indicate mean error rates (with corresponding SEs) for SPT on sizes $(\mathrm{S})$, locations $(\mathrm{L})$, control task $(\mathrm{C})$, reproduction catch $(\mathrm{R})$, and perceptual catch trials $(P)$. Gray bars indicate motor training. Asterisks indicate significant differences $(p<0.05)$.

\section{Materials}

Stimulus materials were made up of visually presented sequences of stimuli. The stimulus items differed from one another in both their size (four sizes) and location (four locations). Each stimulus item was made up of two identical circular forms presented at opposite sides of a virtual circle $\left(11-191^{\circ}, 68-248^{\circ}, 105-295^{\circ}, 164-344^{\circ}\right)$ at $2.5^{\circ}$ of visual angle to the screen center (compare Fig. 3). The circular forms in experimental trials always comprised a larger blue circle (diameter, 35, 28, 21, and $14 \mathrm{~mm}$ ) and a smaller, centrally embedded yellow circle (diameter, 15, 12, 9, and $6 \mathrm{~mm})$.

The circular forms in control trials (compare Fig. 3, bottom) were made up of larger blue or red circles $(21 \mathrm{~mm})$ with centrally embedded red or blue circles $(3.5,6.5,10,13 \mathrm{~mm})$. They were presented at opposite sides of the virtual circle $\left(0-180^{\circ}, 45-225^{\circ}, 90-270^{\circ}, 135-315^{\circ}\right)$ either as twins (nontarget) or as quadruplets (target) (compare Fig. 3, panel 9).

Visual stimuli were presented with VisuaStim (Magnetic Resonance 
Technologies, Northridge, CA) over two small thin film transistor monitors placed directly in front of the eyes, simulating a distance to a normal computer monitor of $\sim 1000 \mathrm{~mm}$.

Trials

One hundred forty-four trials, with a duration of $12 \mathrm{sec}$ and a variable intertrial interval of $1-5$ $\mathrm{sec}$, were presented during the experiment. All trials had the following format (Fig. 3): cue 1 $(600 \mathrm{msec})$, fixation $(600 \mathrm{msec})$, stimulus phase 1 (3600 $\mathrm{msec})$, cue $2(600 \mathrm{msec})$, fixation (600 msec), stimulus phase $2(3600 \mathrm{msec})$, response phase (2000 msec at maximum), and feedback (600 msec). Cue 1 instructed participants as to what property of the presented stimuli would be relevant in the upcoming trial (size, location). During stimulus phase 1, a sequence (made up of three consecutively presented stimuli) was presented twice. This resulted in the presentation of six stimuli for 600 msec each within stimulus phase 1 . Stimulus phase 1 was followed by a second cue $(600$ $\mathrm{msec}$ ), which instructed participants as to the task they would be performing during stimulus phase 2 in the given trial. After stimulus phase 2 , which was made up of six consecutively presented visual stimuli of $600 \mathrm{msec}$ each, participants were asked for their response. Participants were given feedback as to the correctness of their responses on all trials. To enhance the temporal resolution of the blood oxygenation level-dependent (BOLD) signal, variable jitter times of $0,500,1000$, or 1500 msec were inserted, one preceding the first cue, and the other one preceding the second cue.

\section{Tasks}

Participants were instructed and trained in four different experimental tasks and one control task. Which experimental task participants were required to perform was indicated by cue 2 .

SPT trials. In the majority of the experimental trials (72\%; 84 trials), a slightly modified version of the SPT (Schubotz, 1999) was used. In these trials, participants were first instructed by cue 1 as to which stimulus property (size or location) they should attend. In the stimulus phase 1, a sequence consisting of three stimuli was presented twice. In one-half of the trials, the sequential pattern was based on the size of presented stimuli (stimulus phase 1; size sequence encoding). In these trials, the location of the stimuli varied randomly. In the other half of the trials, the sequential pattern was based on the location of stimuli (stimulus phase 1; location sequence encoding). In these trials, the size of the stimuli varied randomly. After stimulus phase 1, participants were instructed by cue 2 to perform a standard SPT (Fig. 3, compare panels 1, 2, 5, and 6), which entailed monitoring the sequences presented in stimulus phase 2 for violations of the sequential pattern presented in stimulus phase 1. Onehalf of the SPT trials contained a sequential violation in stimulus phase 2 (Fig. 3, boldly framed pictures, panels 1 and 5). Sequential violations were constructed by changing the order in which stimuli were presented within the repetition of the sequence in stimulus phase 2 (Fig. 3, compare panels 1 and 5, 10th-12th pictures). Participants were instructed to respond by pressing a button at the end of the violated trials only. Responses were made with the index finger of the hand for which participants had no sensorimotor training, so that right- and left-hand responses were balanced across participants.

Catch trials. Thirty-two catch trials (28\% of the experimental trials) were presented to ensure that participants still remembered the trained sensorimotor mapping they had trained in the pre-experimental training session. To keep the experimental design balanced, one-half of these catch trials were introduced by a cue (cue 1) instructing participants to attend to size, and one-half were introduced by a cue instructing participants to attend to location. Cue 1 was followed, as in the SPT trials, by a repeated sequence of three stimuli. Clearly, only those participants who had trained for the size-related mapping could reproduce the size sequences. Therefore, two different tasks were included in catch trials: (1) participants who had been trained in the relevant stimulus property indicated by cue 1 performed a reproduction task, and (2) participants who had been trained to respond to the stimulus property not indicated by cue 1 performed a perceptual task. The task to be performed was indicated by cue 2 . In reproduction catch trials, participants were instructed to reproduce the observed sequence pattern from stimulus phase 1 via button presses, which they had trained in the pre-experimental session (Fig. 3, compare Rl, Rs, panels 3, 7). After each button press, the corresponding stimulus was presented on the screen and remained visible until the next button press. In perceptual catch trials, participants were instructed to monitor six randomly presented stimuli for a target (Fig. 3, compare panels 4,8). Participants with trained sensorimotor mapping for location therefore performed a perceptual task for trials introduced by a cue 1 instructing attention to size. These participants scanned the stimuli in stimulus phase 2 for a stimulus made up of two objects of different size (compare Fig. 3, Ps, panel 8). Participants with trained sensorimotor mapping for size, on the other hand, performed a perceptual task for trials introduced by a cue 1 instructing attention to location. These participants scanned the stimuli in stimulus phase 2 for a stimulus made up of two objects that were located at points not directly opposite from one another on the virtual circle (compare Fig. 3, Pl, panel 4).

Control trials. Additionally, 28 nonpredictive control trials were introduced to control visual and motor requirements of the SPT. Frequency of 
stimulus presentation was the same as in SPT trials, whereas stimuli were slightly different (see stimuli). A noninstructive second cue was presented in the middle of the trial to keep the overall trial structure identical between SPT and control condition: (1) task cue, (2) target monitoring, (3) dummy cue, (4) target detection. Participants were asked to count the number of targets (four identical geometrical figures on one screen) in the first and in the second part of the trial, respectively. Additionally, nontargets and distractor stimuli that consisted of four geometrical figures of which three were identical and one was deviant were presented but had to be ignored. The response button had to be pressed only if the number of targets presented in the first part of the trial (zero or one) did not match the number of targets presented in the second part of the trial (zero or one), which was the case in $50 \%$ of the control trials.

\section{Training}

Participants took part in a training session 1 week before the experimental session. One group of participants (size group) was trained to associate each of the four different stimulus sizes with a unique button on a four-button response box. The response box was operated with four fingers of the participants' left or right hand, leaving the thumb inactive. Participants learned one of four different sensorimotor mappings (i.e., the learned mapping between stimulus size and button) to ensure that effects (particularly between group effects) were not influenced by unintended mapping compatibility (for one example, compare Fig. 1). In other words, participants within the same group (size group) were trained to respond to a given size with different button presses; whereas one participant learned to map ascending sizes (1-2-3-4) to buttons arranged from left to right (1-2-3-4), another participant learned to associate intermixed sizes (2-1-4-3) to left-to-right buttons (1-2-3-4).

The other group of participants (location group) learned to map the four different possible locations in which stimuli could appear to one unique button on the four-button response box. Again, four fingers of either the left or right hand were used, and the thumb was left inactive. Comparable with participants in the size group, participants in the location group learned four different sensorimotor mappings. After being explicitly instructed as to the stimulus-response correspondences of the sensorimotor mappings, participants practiced the sensorimotor mapping, the four experimental tasks, and the control task until a critical performance level ( $85 \%$ correct responses) was reached in all conditions.

Note that participants with a left-hand mapping will be referred to as left group, whereas participants with a right-hand mapping will be referred to as right group, regardless of the trained stimulus property.

\section{Scanning procedure}

Participants were supine on the scanner bed, with the four fingers of one hand and the index finger of the other hand positioned over the corresponding response buttons. The participants' hands were carefully stabilized, and form-fitting cushions were used to prevent arm, hand, and head motion. To attenuate scanner noise, participants were provided with earplugs. Imaging was performed at $3 \mathrm{~T}$ on a Bruker (Ettingen, Germany) Medspec 30/100 system equipped with the standard birdcage head coil. Slices were positioned parallel to the bicommissural plane (anterior commissure-posterior commissure), with 18 slices (thickness, $5 \mathrm{~mm}$; spacing, $1 \mathrm{~mm}$ ) covering the whole brain. A set of twodimensional anatomical images was acquired for each participant immediately before the functional experiment using a modified driven equilibrium Fourier transformation $($ MDEFT) sequence $(256 \times 256$ pixel matrix). Functional images in plane with the anatomical images were acquired using a single-shot gradient echo-planar (EPI) sequence (echo time, $30 \mathrm{msec}$; pixel matrix, $64 \times 64$; flip angle, $90^{\circ}$; field of view, 192 $\mathrm{mm}$ ). During each trial, eight images were obtained from 18 slices each at a rate of 2 sec per image, resulting in a total of 1312 functional images for each participant, that were obtained in a single run.

\section{Data analysis}

The fMRI data were processed using the software package LIPSIA (Lohmann et al., 2001). Functional data were corrected for motion using a matching metric based on linear correlation. To correct the temporal offset between the slices acquired in one image, a sinc-interpolation algorithm based on the Nyquist-Shannon sampling theorem (Press et al.,
1992) was used. In the preprocessing, low-frequency components of the signal (i.e., baseline drifts) were suppressed by applying a $1 / 120 \mathrm{~Hz}$ highpass filter. A spatial smoothing was performed using a Gaussian kernel of $\sigma=0.6$

To align the functional data slices with a three-dimensional (3D) stereotactic coordinate reference system, a rigid linear registration with six degrees of freedom (three rotational, three translational) was performed. The rotational and translational parameters were acquired on the basis of the MDEFT and EPI-T1 slices to achieve an optimal match between these slices and the individual $3 \mathrm{D}$ reference data set. This $3 \mathrm{D}$ reference data set had been acquired for each participant during a previous scanning session. The MDEFT volume data set with 160 slices and $1 \mathrm{~mm}$ slice thickness was standardized to the Talairach stereotactic space (Talairach and Tournoux, 1988). The rotational and translational parameters were subsequently transformed by linear scaling to a standard size. Transformed parameters were then used to align the functional slices with the stereotactic coordinate system by means of trilinear interpolation, thus generating output data with a spatial resolution of $3 \times 3 \times 3 \mathrm{~mm}\left(27 \mathrm{~mm}^{3}\right)$. This linear normalization process was improved by a subsequent processing step that performed an additional nonlinear normalization (Thirion, 1998).

The statistical analysis was based on a least squares estimation using the general linear model for serially autocorrelated observations (Friston, 1994; Friston et al., 1995a,b; Worsley and Friston, 1995). The design matrix was generated with a boxcar function, convolved with the hemodynamic response function, including a delay of $6 \mathrm{sec}$. The analyzed epoch comprised the first $3600 \mathrm{msec}$ after the onset of the first stimulus of SPT and control trials in which participants responded correctly. The model equation, including the observation data, the design matrix, and the error term, was convolved with a Gaussian kernel of dispersion of 4 sec full width at half maximum (Worsley and Friston, 1995). In the following, contrast images (i.e., estimates of the raw-score differences between SPT and control task) were generated for each participant. Because the individual functional data sets were all aligned to the same stereotactic reference space, group analyses were performed subsequently. The single-participant contrast images were compared voxelwise using two-sample $t$ tests to examine the hypothesis that the mean contrasts differed between groups. The resulting $t$ values were transformed so that the resulting image contained $Z$-scores indicating the degree of significance of the group difference. To minimize the probability of false positives (type I error), only voxels with a $Z$-score of $>3.09$ $\left(p<0.001\right.$, uncorrected) in a cluster with a volume of $>225 \mathrm{~mm}^{3}$ (eight contiguous voxels) were considered as activated and depicted in figures. However, lowering or raising the cluster threshold to a volume of 135 or $270 \mathrm{~mm}^{3}$, respectively, did not influence the effects in premotor cortex.

To perform a region-of-interest analysis, percentage BOLD signal change (PSC) was extracted from selected voxels $(3 \times 3 \times 3 \mathrm{~mm})$, as specified in Results. To eliminate unspecific effects (e.g., of visual stimulation and response mode), the PSC for the control task was subtracted from the PSC obtained for the serial prediction task before defining maximal PSC.

For additional individual peak localization, the contrast between SPT and control task was calculated using the $t$ statistic. Subsequently, $t$ values were transformed into $Z$-scores, and individual peaks of activation (SPT greater than control task) within a prespecified premotor space were extracted from $Z$-maps. We used the following extensions for the lateral premotor space: coronal coordinate, $x \geq 20$ or $x \leq-20$; sagittal coordinate, $-12 \geq y \geq 12$; and axial coordinate, $z \geq 5$. Subsequently, discriminant analyses were performed on the locations (axial and sagittal) of individual activation peaks for left and right hemisphere separately.

\section{Results}

\section{Behavioral results}

Mean error rates were computed for each experimental condition. Influences of task (size SPT, location SPT, control task), motor effector (left vs right hand), and trained property (size vs location) on behavioral performance were investigated by means of repeated-measures ANOVA. If required, Greenhouse-Geisser 
Table 1. Influence of the attended stimulus property

\begin{tabular}{|c|c|c|c|c|c|}
\hline Area & $x$ & $y$ & $Z$ & $\mathrm{~mm}^{3}$ & $Z$ \\
\hline \multicolumn{6}{|l|}{ SPT without motor training versus control } \\
\hline \multicolumn{6}{|l|}{ Size group $>$ location group } \\
\hline \multicolumn{6}{|l|}{ L inferior precentral sulcus-middle } \\
\hline frontal gyrus & -40 & 8 & 32 & 837 & 4.2 \\
\hline L intraparietal sulcus-inferior parietal lobule & -28 & -64 & 41 & 923 & 4.3 \\
\hline L middle occipital gyrus & -22 & -100 & 3 & 4378 & 4.5 \\
\hline R fusiform gyrus & 28 & -76 & -6 & 1481 & 4.0 \\
\hline$R$ cerebellum & 5 & -76 & -21 & 385 & 4.1 \\
\hline \multicolumn{6}{|l|}{ Location group $>$ size group } \\
\hline \multicolumn{6}{|l|}{ R superior precentral sulcus-superior } \\
\hline frontal sulcus & 25 & -10 & 50 & 3256 & 5.2 \\
\hline \multicolumn{6}{|l|}{ L superior precentral sulcus-superior } \\
\hline frontal sulcus & -22 & -7 & 56 & 2255 & 5.7 \\
\hline L superior frontal sulcus & -20 & 26 & 44 & 273 & 3.8 \\
\hline R inferior parietal lobule & 56 & -34 & 44 & 1811 & 4.4 \\
\hline R superior parietal lobule & 29 & -43 & 53 & 828 & 4.0 \\
\hline \multicolumn{6}{|l|}{ SPT with motor training versus control } \\
\hline \multicolumn{6}{|l|}{ Location group $>$ size group } \\
\hline L postcentral sulcus-superior parietal lobule & -11 & -43 & 56 & 291 & 3.8 \\
\hline
\end{tabular}

Anatomical specification, hemisphere, Talairach coordinates $(x, y, z)$, volume (in cubed millimeters), and maximal $Z$-scores of brain areas differing significantly between participants attending to sizes and participants attending to locations (contrasted against target monitoring control task). L, Left hemisphere; R, right hemisphere.

correction was applied. Across all conditions, participants with training for size did not make significantly more errors than those with training for location (no main effect trained property, $\left.F_{(1,28)}=0.02 ; p=0.905\right)$. Likewise, participants with left-hand training did not make significantly more errors than those with right-hand training (no main effect motor effector, $\left.F_{(1,28)}=0.77 ; p=0.389\right)$. However, tasks differed in terms of error rates (main effect task, $F_{(1.62,56)}=12.21 ; p<$ $0.001)$, and a significant task by trained property interaction $\left(F_{(1.62,56)}=5.03 ; p=0.016\right)$ prompted a closer inspection by means of post hoc $t$ tests. Participants with training for size (size group) made significantly more errors in the control task $(13.2 \%)$ compared with serial prediction tasks on size $(7.0 \% ; p=0.013)$ and location $(6.0 \% ; p=0.004)$. Between the latter two tasks, error rates did not differ significantly ( $p=0.443$ ) (Fig. 3, bottom right). In contrast, participants with training for location (location group) made significantly less errors in the serial prediction task on location $(4.5 \%)$ compared with both the control task $(9.6 \% ; p=0.001)$ and serial prediction task on size $(11.2 \% ; p=$ $0.001)$. Error rates did not differ significantly between control task and size SPT ( $p=0.485$ ), as depicted in Figure 3 (top right). No other significant interactions were observed. Performance in catch trials is depicted (Fig. 3, bars R and P) for descriptive purposes only and was not subjected to additional analyses.

\section{Imaging results}

In the following, we focus on BOLD effects within the lateral premotor cortex (other activations are listed in Table 1). Effects of the to-be-attended stimulus property were analyzed by comparing activation in participants attending to size sequences to activation in participants attending to location sequences. According to our hypotheses, we computed two types of contrasts. Contrasts for trials without motor training reflect differences between size SPT in the location group and location SPT in the size group. Contrasts for trials with motor training reflect differences between size SPT in the size group and location SPT in the location group. Effects of the motor effector were analyzed by comparing activation for SPT with motor training in the left and right group. Unspecific effects of visual stimulation, response mode, and error detection were eliminated by subtracting activation of the control condition before computing group differences.

\section{Attended stimulus property}

Regarding trials without motor training, we found pronounced differences in activation in the lateral premotor cortex. Whereas attending to size led to significantly stronger activation within the left ventral PM (inferior precentral sulcus bordering posterior middle frontal gyrus), attending to location activated the bilateral dorsal PM more strongly (Fig. 4, Table1).

This finding was supported by discriminant analyses on axial and sagittal location of individual peaks with to-be-attended stimulus property (size vs location) as group variable. In left PM, axial location of peaks (Fig. 4, top left) discriminated best between participants attending to size and participants attending to location without motor training $\left(\lambda_{1}=0.68 ; p=0.001\right)$. Because $80.6 \%$ of the participants were classified correctly (critical chance level $\approx 65 \%$; one-tailed $p=0.05$ ) for left PM, the predicted differentiation (i.e., dorsal peaks for location sequences and ventral peaks for size sequences) was confirmed. In contrast, activation peaks in the right hemisphere were located in dorsal PM (Fig. 4, top right) and did not differ significantly in axial location between participants attending to size sequences and participants attending to location sequences without motor training. However, groups could be discriminated by the sagittal location $\left(\lambda_{1}=\right.$ $0.70 ; p=0.002$; correct, $76.7 \%)$. Thus, attending to size sequences without motor training led to more anterior peaks in right dorsal PM than attending to location sequences without motor training.

In contrast, regarding sequences with motor training (Fig. 4, middle, Table 1), group comparisons revealed no significant differences in premotor activation (i.e., differential effects of to-beattended stimulus property on premotor activation were not observed).

\section{Motor effector}

Comparing activation in participants with left- and right-hand training revealed differences in the respective contralateral sensorimotor cortex during SPT with motor training. Moreover, as listed in Table 2, motor training for the left hand was associated with stronger activation in the vicinity of right inferior precentral sulcus and posterior middle frontal gyrus (anterior dorsal PM). Maximal percentage BOLD signal change (PSC) was extracted from these three sites. Repeated-measures ANOVAs were performed with within-subject factor task (SPT with vs without motor training) and between-subject factors trained property (size vs location) and motor effector (left vs right hand). Regarding PSC extracted from right anterior dorsal PM, main effects for motor effector $\left(F_{(1,28)}=6.34 ; p=0.018\right)$ (compare Fig. 5) and task $\left(F_{(1,28)}=6.31 ; p=0.018\right)$ were revealed. Importantly, the left group showed greater PSC at this site than the right group during attending to both sequences with and without motor training. In contrast, ANOVAs on PSC extracted from the sensorimotor cortex (compare Fig. 5) revealed significant task by motor effector interactions (left hemisphere, $F_{(1,28)}=12.08, p=$ 0.002 ; right hemisphere, $\left.F_{(1,28)}=21.84, p<0.001\right)$. Serial prediction task with motor training elicited stronger PSC than serial prediction task without motor training in contralateral sensorimotor cortex only. Importantly, the motor effector influenced the PSC in the sensorimotor cortex only during SPT with motor training. 


\section{Target properties}

Individual activation peaks for SPT with motor training were located in dorsal PM in both hemispheres (Fig. 4, compare middle left and right), and participants attending to size sequences could not be discriminated from those attending to location sequences (correct, 53.1-62.5\%; corresponding to chance level). To gain a more detailed insight, maximal percentage BOLD signal change was extracted from the voxel in left and right dorsal PM differing maximally between size and location group during SPT without motor training (Fig. 4, bottom). Repeated-measures ANOVAs were performed with within-subject factor task (SPT with vs without motor training) and between-subject factors trained property (size vs location) and motor effector (left vs right hand). The percentage signal change in left and right dorsal PM was not affected by the motor effector. As depicted in the bottom panel of Figure 4, interactions of trained property and task emerged in both left dorsal PM $\left(F_{(1,28)}=8.51 ; p=\right.$ $0.007)$ and right dorsal PM $\left(F_{(1,28)}=\right.$ 31.74; $p<0.001)$. These interactions can be decomposed in between-group and within-group components. Between-group differences in PSC were observed for SPT without motor training (location greater than size) but not for SPT with motor training. Within-group differences in PSC were observed only in the location group (compare Fig. 4, bottom, dashed brackets). In these participants, SPT with motor training (i.e., SPT on locations) elicited stronger PSC (left dorsal PM, 0.58\%; right dorsal PM, $0.35 \%$ ) than SPT without motor training (i.e., SPT on sizes) (left dorsal PM, 0.22\%, $p<0.001$; right dorsal PM, 0.11\%, $p<$ $0.001)$. Hence, in dorsal PM, the PSC for size SPT with motor training increased to the level observed for location SPT with and without motor training.

\section{Discussion}

The objective of the present fMRI study was to investigate if and how premotor correlates of object (size) and spatial (location) attention change in the presence of a nonstandard mapping. In particular, we aimed to find out which parameter of the nonstandard mapping (i.e., motor effector or target property) causes changes in the premotor activation pattern. First, results replicated property-specific modulations of premotor activation when participants merely attended to temporal order information provided by abstract stimulus sequences (Schubotz and von Cramon, 2001). This attentional modulation of premotor activation did not occur when a nonstandard mapping had been acquired beforehand. Second, trained hand did not cause lateralization toward the contralateral PM. Third, individual peaks of activation converged on dorsal PM, no matter to which stimulus property participants attended. The findings have
Size - Location: SPT without motor training
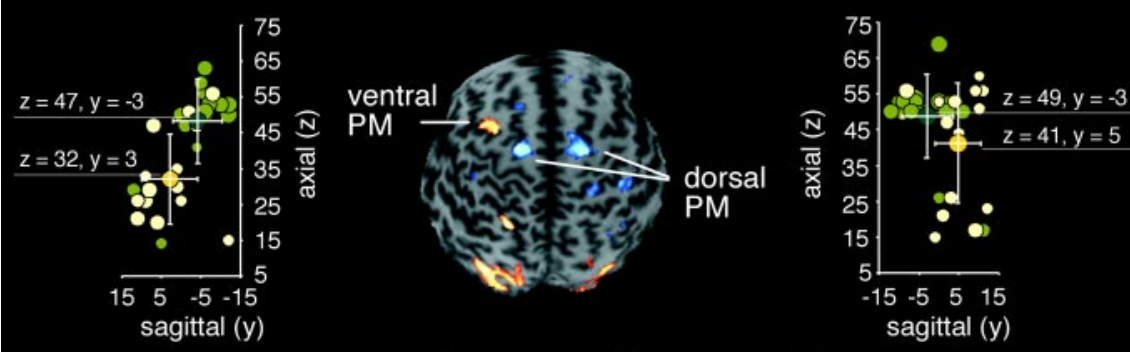

Size - Location: SPT with motor training
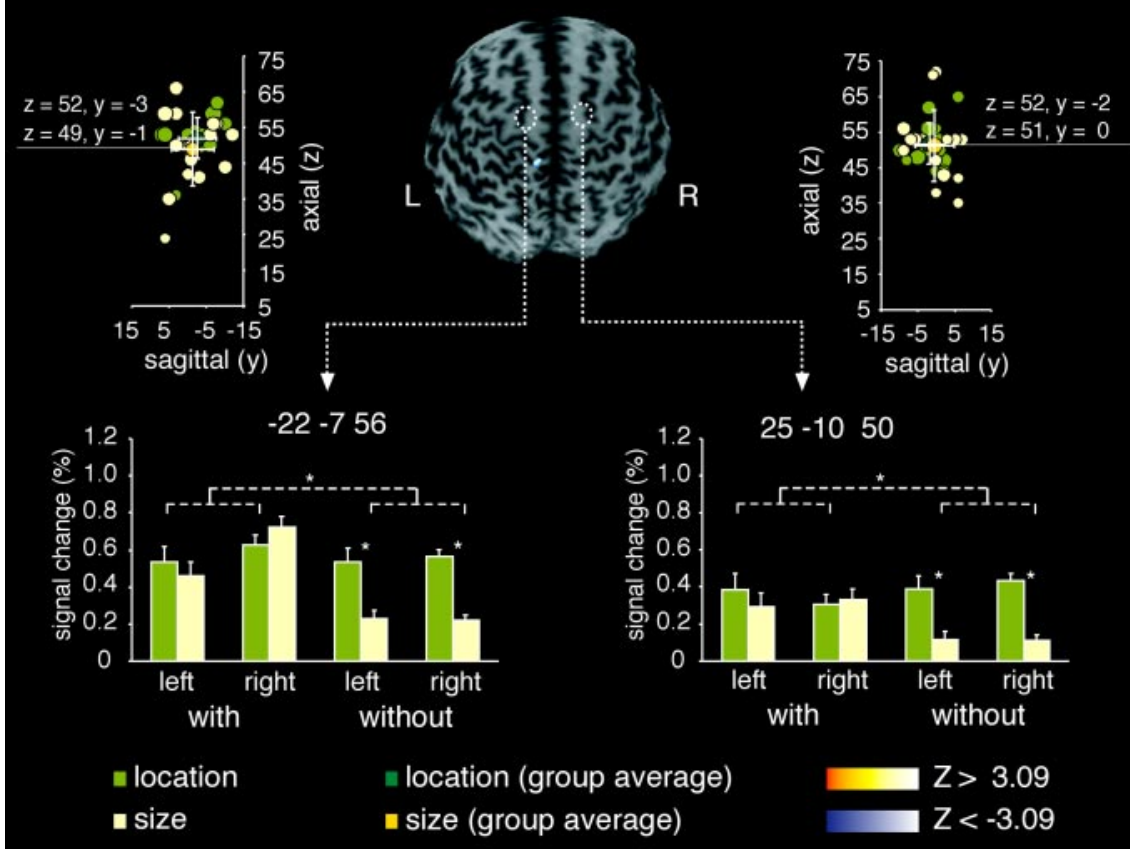

Figure 4. Differential modulation of PM by to-be-attended stimulus property vanishes in the presence of a nonstandard mapping. Top and middle, Results of second level $t$ tests (attended property, size vs location) for SPT without (top) and with motor (light yellow) and location SPT (light green) are depicted separately for the left (outer left panels) and right hemisphere (oute , The maximal percentage signal change extracted from two dorsal PM peaks of activation (compare top panel) is depicted to SPT with motor training, whereas the right four bars refer to SPT without motor training. Colors indicate the currently to-beattended stimulus property, not the group identity. For instance, a participant labeled "left, yellow, with motor training," corresponding to size SPT also occurs labeled "left, green, without motor training," corresponding to location SPT. Terms left and right refer to the training hand, not to the response hand, which would be the contralateral one. Significant between-group differences (size SPT vs location SPT) are indicated by asterisks ( $p<0.05$ ), whereas significant within-group differences (SPT with motor training vs SPT without) are indicated by asterisks on dashed brackets $(p<0.05)$. L, Left hemisphere; $R$, right hemisphere.

\section{Table 2. Influence of the motor effector}

\begin{tabular}{|c|c|c|c|c|c|}
\hline Area & $x$ & $y$ & $z$ & $\mathrm{~mm}^{3}$ & Z \\
\hline \multicolumn{6}{|l|}{ SPT with motor training versus control } \\
\hline \multicolumn{6}{|l|}{ Left group $>$ right group } \\
\hline R inferior precentral sulcus-middle frontal gyrus & 41 & 5 & 47 & 229 & 4.0 \\
\hline R postcentral gyrus & 34 & -25 & 50 & 1681 & 4.5 \\
\hline \multicolumn{6}{|l|}{ Right group $>$ left group } \\
\hline L postcentral gyrus & -32 & -22 & 44 & 1347 & 4.2 \\
\hline L postcentral gyrus & -50 & -16 & 30 & 433 & 4.2 \\
\hline R cerebellum & 20 & -46 & -24 & 502 & 3.9 \\
\hline
\end{tabular}

Anatomical specification, hemisphere, Talairach coordinates $(x, y, z)$, volume (in cubed millimeters), and maximal $Z$-scores of brain areas differing significantly between participants with left-hand training and participants with right-hand training (contrasted against target monitoring control task). L, Left hemisphere; $\mathrm{R}$, right hemisphere. 


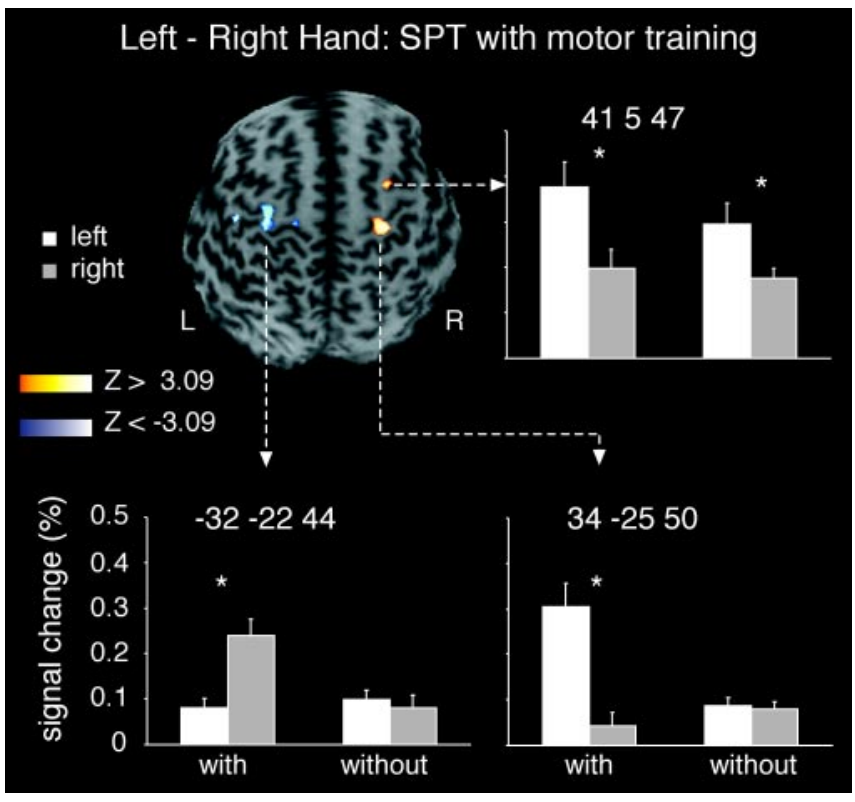

Figure 5. Influence of the motor effector. Results of second level $t$ test (left vs right hand) for SPT with motor training are depicted on an intersection flatbrain. The maximal percentage signal changes were extracted from the premotor (top right) and sensorimotor voxels (bottom), which differed maximally between participants trained with the left hand (white bars) and those trained with the right hand (gray bars). Within each graph, PSC is depicted for SPT with and without motor training. Significant differences are indicated by asterisks. L, Left hemisphere; $R$, right hemisphere. Error bars represent $S E$.

important implications for the question of how nonstandard mappings are implemented in PM. Specifically, they suggest that premotor contribution to nonstandard mapping consists in coding of target properties rather than motor effectors. The motor effector of the acquired mapping, in turn, was found to cause differential effects in the central sulcus area according to the typical contralateral pattern.

According to the first hypothesis, which was based on previous fMRI studies (Schubotz and von Cramon, 2001; Schubotz et al., 2003), we expected different foci of activation within the premotor cortex for attending to size sequences (ventral PM) compared with attending to location sequences (dorsal PM). The present findings confirmed this hypothesis in the absence of a motor training. This replicates a property-specific modulation of premotor activation during attending to visual sequences. The specific correspondence between to-be-predicted stimulus properties and activated lateral premotor areas may be explained by a preference of reaching areas for spatial targets and one of grasping areas for object targets (habitual pragmatic body map) (Schubotz and von Cramon, 2003; Schubotz et al., 2003). This account may more generally explain property-specific modulations of the human lateral premotor cortex, as reported in recent imaging studies using other experimental paradigms. Accordingly, as shown by a meta-analysis (Schubotz and von Cramon, 2003), similar areas within dorsal PM are engaged for both arm movements and spatial attention and likewise, within ventral PM for both hand movements and object attention. A new finding is that the typical premotor activation pattern was not observed when participants had acquired a nonstandard mapping for object and spatial stimuli. Hence, a first conclusion we can draw is that having acquired a nonstandard mapping changes the way attended stimulus properties are represented in PM. It has to be considered that participants might have construed "global" ob- jects in the location task (e.g., by connecting the two "local" objects presented on one screen with a virtual bar). Even in this case, however, the location task could only be performed successfully when the spatial properties of such a global object were attended to, and the local object properties (size) were ignored. In the location task, only locations were sequentially ordered, whereas size varied randomly and independently from the locations.

According to the second hypothesis, premotor correlates should differ between the groups trained with the left or right hand if the motor effector of the acquired mapping was reflected in PM. The present findings do not support this hypothesis, because the influence of the motor effector on premotor activation was restricted to a general group effect. This is in line with a previous study (Rijntjes et al., 1999), showing that writing one's signature with either hand or toe relies on the same premotor areas, indicating that the somatotopy in PM is defined functionally, rather than on the basis of anatomical representations. In contrast, activation of contralateral central sulcus area, including primary sensorimotor cortex, differed between left and right group during SPT with motor training only. One might argue that this activation pattern resulted from a not instructed overt or covert (imagined) coproduction of the attended stimulus sequence. It has been demonstrated that execution as well as imagery (although to a lesser extent) of finger movements involve contralateral sensorimotor areas (Porro et al., 1996, 2000; Grafton et al., 1998; Ehrsson et al., 2003; Nair et al., 2003). It is typically reported that either both primary motor and premotor areas (Porro et al., 1996; Lotze et al., 1999; Boecker et al., 2002; Stippich et al., 2002; Ehrsson et al., 2003) or premotor areas alone (Decety et al., 1994; Stephan et al., 1995; Deiber et al., 1998; Dechent et al., 2004) are engaged during motor imagery. Therefore, we suggest that covert coproduction does not very plausibly account for the present results. An alternative explanation would be that primary motor areas code that part of the nonstandard mapping that is important for motor output (i.e., the motor effector).

According to the third hypothesis, premotor correlates of object and spatial attention should converge on dorsal PM if the target property of the acquired mapping is reflected in PM. Correspondingly, direct task contrast did not reveal any significant differences between attending to size and attending to location in the presence of a nonstandard mapping. Individual peaks of premotor activation were located within dorsal premotor areas both in the size group attending to size sequences and in the location group attending to location sequences, respectively. Moreover, signal intensity increased in dorsal PM for size SPT with motor training up to the level observed for location SPT with and without motor training. Note that both size sequences and location sequences with motor training had to be translated into spatial sequences as a result of the horizontal array of the response buttons. Because in the absence of a motor training, dorsal PM was observed to be specifically engaged for location but not size sequences, the convergence of activations on dorsal PM in the presence of a motor training is most parsimoniously explained by the spatial target properties. Dorsal PM activations have been reported in visuomanual serial learning tasks using spatial response arrangements (Honda et al., 1998; Harrington et al., 2000; Boecker et al., 2002; Grafton et al., 2002; Haslinger et al., 2002). These studies found the dorsal PM to be particularly engaged in complex compared with simple sequences. Although the dorsal PM has therefore often been related to sequential complexity in general, this interpretation is rendered implausible by the finding that increasing sequential complexity in nonspatial sequences, 
such as size-based and pitch sequences, particularly engages ventral premotor areas (Schubotz and von Cramon, 2002a,b). Moreover, dorsal PM engagement in spatial processing has been reported more generally in nonsequential paradigms in humans (Griffiths et al., 2000; Nobre et al., 2000; Lamm et al., 2001; Pochon et al., 2001; Vingerhoets et al., 2002; Handy et al., 2003). This is in line with a contribution of nonhuman primate dorsal PM to spatial tasks in general, not only sequential ones (Kubota and Hamada, 1978; Passingham, 1987; Boussaoud and Wise, 1993; Shen and Alexander, 1997; Boussaoud, 2001; Lebedev and Wise, 2001).

Note, however, that on the basis of the present study alone, we cannot decide whether the convergence of activation on dorsal PM reflects coding of intrinsic or extrinsic spatial properties of the response (i.e., the fingers or the response buttons, respectively). For ventral PM, a series of recent studies in monkeys point to extrinsic-like rather than intrinsic-like coding (Kakei et al., 1999, 2001, 2003). Moreover, the representation of the fingers has been located within ventral PM rather than in dorsal PM (Godschalk et al., 1995; Preuss et al., 1996). Together, these findings support the notion that in the present study, dorsal PM activation reflects coding of the spatial arrangement of the response buttons rather than the motor effector.

In some studies, dorsal PM has been reported to be specifically engaged in nonstandard mapping, referring to spatially incompatible mappings, in contrast to spatially compatible ones (Iacoboni et al., 1998; Bischoff-Grethe et al., 2004). Because spatial mapping is inherent to both compatible and incompatible mappings in these tasks, spatial mapping alone cannot account for stronger dorsal PM activation in incompatible mappings. However, dorsal PM effects reported in these studies can also be explained by the requirement of transforming arbitrary cues into spatial properties. Involvement of dorsal PM in spatial transformation has in turn been reported in monkeys (Ohbayashi et al., 2003), as well as in humans (Iacoboni et al., 1998; Harrington et al., 2000; Binkofski et al., 2003). Moreover, it has been shown that dorsal PM covaries with the complexity of the spatial response structure (spatial transformations) rather than the motor structure (involved fingers) of visually guided sequences (Harrington et al., 2000). Together, the data imply that dorsal PM activation can result either from spatial representations in a purely attentional setting, as well as from rule-based spatial transformations as required by most arbitrary sensorimotor mappings. Although arbitrariness is often considered to account for dorsal PM activation in nonstandard mappings, all available studies on that issue employ spatial transformations and therefore confound both features.

Finally, we would like to outline how the present findings enhance the understanding of PM contribution to attentional processes. We started with the hypothesis that attended stimulus properties are represented according to a default sensorimotor association, linking object-specific properties to the ventral PM and spatial properties to the dorsal PM (Schubotz et al., 2003). The present study confirms this notion. However, it also shows that in the presence of a concretely defined sensorimotor association, activation in PM in response to object or spatial attention changes, and converges on dorsal PM. We propose this convergence to reflect that object-specific and spatial stimulus properties, in practical terms, have become identical: now the relevant feature of the attended stimuli is the spatial arrangement of the response buttons. In other words, acquiring a nonstandard mapping appears to shift relevance from stimulus to target properties.

An issue of future research would be to test this account with nonspatial target properties (cf. Brasted et al., 2003). This is par- ticularly indicated because dorsal PM lesions correlate with impairments in go-nogo task learning (Petrides, 1986). Moreover, to prove fully that dorsal PM activity reflects target properties, one could use a mapping transfer paradigm, as has been used in studies on motor sequence learning. Transferring between different mappings within the same target property (e.g., different spatial mappings) has been used by Bapi et al. (2000). Applied to the present study, such a transfer between different spatial mappings would be expected to transiently enhance dorsal PM activation. More appropriate for the present purpose, however, would be to introduce an effector-effector transfer as has been used, for example, by Grafton et al. (1998). If dorsal PM reflects spatial target properties of the acquired mapping, one would expect that a transfer between, for example, manual and arm responses would not change the effect found in the present study. A final alternative would be to use a transfer between different target properties, as, for example, from spatial targets to object-related targets. In this case, we would expect a shift of activation from dorsal to ventral PM.

\section{Conclusion}

PM activation in attentional tasks significantly depends on the attended stimulus property. The present findings show that this dependency changes as a consequence of an arbitrary sensorimotor training applied on the same stimulus material. In that case, PM activation can neither be distinguished by the attended stimulus property nor by the motor effector of the trained response. It rather seems to reflect properties of the acquired response. To pick up the previous example, in the eyes of the car driver, one could say, to attend to the redness of the traffic light is synonymous to attending to the brake pedal.

\section{References}

Bapi RS, Doya K, Harner AM (2000) Evidence for effector independent and dependent representations and their differential time course of acquisition during motor sequence learning. Exp Brain Res 132:149-162.

Binkofski F, Butler A, Buccino G, Heide W, Fink G, Freund HJ, Seitz RJ (2003) Mirror apraxia affects the peripersonal mirror space. A combined lesion and cerebral activation study. Exp Brain Res 153:210-219.

Bischoff-Grethe A, Goedert KM, Willingham DT, Grafton ST (2004) Neural substrates of response-based sequence learning using fMRI. J Cogn Neurosci 16:127-138.

Boecker H, Ceballos-Baumann AO, Bartenstein P, Dagher A, Forster K, Haslinger B, Brooks DJ, Schwaiger M, Conrad B (2002) A H(2)(15)O positron emission tomography study on mental imagery of movement sequences-the effect of modulating sequence length and direction. NeuroImage 17:999-1009.

Boussaoud D (2001) Attention versus intention in the primate premotor cortex. NeuroImage 14:S40-45.

Boussaoud D, Wise SP (1993) Primate frontal cortex: neuronal activity following attentional versus intentional cues. Exp Brain Res 95:15-27.

Brasted PJ, Bussey TJ, Murray EA, Wise SP (2003) Role of the hippocampal system in associative learning beyond the spatial domain. Brain 126:1202-1223.

Decety J, Perani D, Jeannerod M, Bettinardi V, Tadary B, Woods R, Mazziotta JC, Fazio F (1994) Mapping motor representations with positron emission tomography. Nature 371:600-602.

Dechent P, Merboldt KD, Frahm J (2004) Is the human primary motor cortex involved in motor imagery? Brain Res Cogn Brain Res 19:138-144.

Deiber MP, Ibanez V, Honda M, Sadato N, Raman R, Hallett M (1998) Cerebral processes related to visuomotor imagery and generation of simple finger movements studied with positron emission tomography. NeuroImage 7:73-85.

Ehrsson HH, Geyer S, Naito E (2003) Imagery of voluntary movement of fingers, toes, and tongue activates corresponding body-part-specific motor representations. J Neurophysiol 90:3304-3316.

Friston KJ (1994) Statistical parametric mapping. In: Functional neuroimaging: technical foundations (Thatcher RW, Hallet M, Zeffiro T, John ER, Huerta M, eds), pp 79-93. San Diego: Academic. 
Friston KJ, Holmes AP, Poline JB, Grasby PJ, Williams SC, Frackowiak RS, Turner R (1995a) Analysis of fMRI time-series revisited. NeuroImage 2:45-53.

Friston KJ, Holmes AP, Worsley KJ, Poline JP, Frith CD, Frackowiak RS (1995b) Statistical parametric maps in functional imaging: a general linear approach. Hum Brain Mapp 2:189-210.

Godschalk M, Mitz AR, van Duin B, van der Burg H (1995) Somatotopy of monkey premotor cortex examined with microstimulation. Neurosci Res 23:269-279.

Grafton ST, Hazeltine E, Ivry RB (1998) Abstract and effector-specific representations of motor sequences identified with PET. J Neurosci 18:9420-9428.

Grafton ST, Hazeltine E, Ivry RB (2002) Motor sequence learning with the nondominant left hand. A PET functional imaging study. Exp Brain Res 146:369-378

Griffiths TD, Green GG, Rees A, Rees G (2000) Human brain areas involved in the analysis of auditory movement. Hum Brain Mapp 9:72-80.

Handy TC, Grafton ST, Shroff NM, Ketay S, Gazzaniga MS (2003) Graspable objects grab attention when the potential for action is recognized. Nat Neurosci 6:421-427.

Harrington DL, Rao SM, Haaland KY, Bobholz JA, Mayer AR, Binder JR, Cox RW (2000) Specialized neural systems underlying representations of sequential movements. J Cogn Neurosci 12:56-77.

Haslinger B, Erhard P, Weilke F, Ceballos-Baumann AO, Bartenstein P, Grafin von Einsiedel H, Schwaiger M, Conrad B, Boecker H (2002) The role of lateral premotor-cerebellar-parietal circuits in motor sequence control: a parametric fMRI study. Brain Res Cogn Brain Res 13:159-168.

Honda M, Deiber MP, Ibanez V, Pascual-Leone A, Zhuang P, Hallett M (1998) Dynamic cortical involvement in implicit and explicit motor sequence learning. A PET study. Brain 121:2159-2173.

Hoshi E, Tanji J (2004) Functional specialization in dorsal and ventral premotor areas. Prog Brain Res 143:507-511.

Iacoboni M, Woods RP, Mazziotta JC (1998) Bimodal (auditory and visual) left frontoparietal circuitry for sensorimotor integration and sensorimotor learning. Brain 121:2135-2143.

Kakei S, Hoffman DS, Strick PL (1999) Muscle and movement representations in the primary motor cortex. Science 285:2136-2139.

Kakei S, Hoffman DS, Strick PL (2001) Direction of action is represented in the ventral premotor cortex. Nat Neurosci 4:1020-1025.

Kakei S, Hoffman DS, Strick PL (2003) Sensorimotor transformations in cortical motor areas. Neurosci Res 46:1-10.

Kubota K, Hamada J (1978) Visual tracking and neuron activity in the postarcuate area in monkeys. J Physiol (Lond) 74:297-312.

Lamm C, Windischberger C, Leodolter U, Moser E, Bauer H (2001) Evidence for premotor cortex activity during dynamic visuospatial imagery from single-trial functional magnetic resonance imaging and eventrelated slow cortical potentials. NeuroImage 14:268-283.

Lebedev MA, Wise SP (2001) Tuning for the orientation of spatial attention in dorsal premotor cortex. Eur J Neurosci 13:1002-1008.

Lohmann G, Muller K, Bosch V, Mentzel H, Hessler S, Chen L, Zysset S, von Cramon DY (2001) LIPSIA-a new software system for the evaluation of functional magnetic resonance images of the human brain. Comput Med Imaging Graph 25:449-457.

Lohmann G, Schubotz RI, von Cramon DY (2002) Visualization of fMRI data using coronal flatmaps. NeuroImage 16:S476.

Lotze M, Montoya P, Erb M, Hulsmann E, Flor H, Klose U, Birbaumer N, Grodd W (1999) Activation of cortical and cerebellar motor areas during executed and imagined hand movements: an fMRI study. J Cogn Neurosci 11:491-501.

Luppino G, Murata A, Govoni P, Matelli M (1999) Largely segregated parietofrontal connections linking rostral intraparietal cortex (areas AIP and VIP) and the ventral premotor cortex (areas F5 and F4). Exp Brain Res 128:181-187.

Mitz AR, Godschalk M, Wise SP (1991) Learning-dependent neuronal activity in the premotor cortex: activity during the acquisition of conditional motor associations. J Neurosci 11:1855-1872.

Murray EA, Bussey TJ, Wise SP (2000) Role of prefrontal cortex in a network for arbitrary visuomotor mapping. Exp Brain Res 133:114-129.

Nair DG, Purcott KL, Fuchs A, Steinberg F, Kelso JA (2003) Cortical and cerebellar activity of the human brain during imagined and executed unimanual and bimanual action sequences: a functional MRI study. Brain Res Cogn Brain Res 15:250-260.
Nobre AC, Gitelman DR, Dias EC, Mesulam MM (2000) Covert visual spatial orienting and saccades: overlapping neural systems. NeuroImage 11:210-216.

Ohbayashi M, Ohki K, Miyashita Y (2003) Conversion of working memory to motor sequence in the monkey premotor cortex. Science 301:233-236.

Passingham RE (1987) Two cortical systems for directing movement. Ciba Found Symp 132:151-164.

Petrides M (1986) The effect of periarcuate lesions in the monkey on the performance of symmetrically and asymmetrically reinforced visual and auditory go, no-go tasks. J Neurosci 6:2054-2063.

Pochon JB, Levy R, Poline JB, Crozier S, Lehericy S, Pillon B, Deweer B, Le Bihan D, Dubois B (2001) The role of dorsolateral prefrontal cortex in the preparation of forthcoming actions: an fMRI study. Cereb Cortex 11:260-266.

Porro CA, Francescato MP, Cettolo V, Diamond ME, Baraldi P, Zuiani C, Bazzocchi M, di Prampero PE (1996) Primary motor and sensory cortex activation during motor performance and motor imagery: a functional magnetic resonance imaging study. J Neurosci 16:7688-7698.

Porro CA, Cettolo V, Francescato MP, Baraldi P (2000) Ipsilateral involvement of primary motor cortex during motor imagery. Eur J Neurosci 12:3059-3063.

Press WH, Teukolsky SA, Vetterling WT, Flannery BP (1992) Numerical recipes in C, Ed 2. Cambridge, UK: Cambridge UP.

Preuss TM, Stepniewska I, Kaas JH (1996) Movement representation in the dorsal and ventral premotor areas of owl monkeys: a microstimulation study. J Comp Neurol 371:649-676.

Rijntjes M, Dettmers C, Buchel C, Kiebel S, Frackowiak RS, Weiller C (1999) A blueprint for movement: functional and anatomical representations in the human motor system. J Neurosci 19:8043-8048.

Rizzolatti G, Luppino G (2001) The cortical motor system. Neuron 31:889-901.

Schubotz R (1999) Instruction differentiates the processing of temporal and spatial sequential patterns: evidence from slow wave activity in humans. Neurosci Lett 265:1-4.

Schubotz RI, von Cramon DY (2001) Functional organization of the lateral premotor cortex: fMRI reveals different regions activated by anticipation of object properties, location and speed. Brain Res Cogn Brain Res 11:97-112.

Schubotz RI, von Cramon DY (2002a) Predicting perceptual events activates corresponding motor schemes in lateral premotor cortex: an fMRI study. NeuroImage 15:787-796.

Schubotz RI, von Cramon DY (2002b) A blueprint for target motion: fMRI reveals perceived sequential complexity to modulate premotor cortex. NeuroImage 16:920-935.

Schubotz RI, von Cramon DY (2002c) Dynamic patterns make the premotor cortex interested in objects: influence of stimulus and task revealed by fMRI. Brain Res Cogn Brain Res 14:357-369.

Schubotz RI, von Cramon DY (2003) Functional-anatomical concepts of human premotor cortex: evidence from fMRI and PET studies. NeuroImage 20:S120-S131.

Schubotz RI, von Cramon DY, Lohmann G (2003) Auditory what, where, and when: a sensory somatotopy in lateral premotor cortex. NeuroImage 20:173-185.

Shen L, Alexander GE (1997) Preferential representation of instructed target location versus limb trajectory in dorsal premotor area. J Neurophysiol 77:1195-1212.

Stephan KM, Fink GR, Passingham RE, Silbersweig D, Ceballos-Baumann AO, Frith CD, Frackowiak RS (1995) Functional anatomy of the mental representation of upper extremity movements in healthy subjects. J Neurophysiol 73:373-386.

Stippich C, Ochmann H, Sartor K (2002) Somatotopic mapping of the human primary sensorimotor cortex during motor imagery and motor execution by functional magnetic resonance imaging. Neurosci Lett 331:50-54.

Talairach J, Tournoux P (1988) Co-planar stereotaxic atlas of the human brain. Stuttgart, Germany: Thieme.

Thirion JP (1998) Image matching as a diffusion process: an analogy with Maxwell's demons. Med Image Anal 2:243-260.

Vingerhoets G, de Lange FP, Vandemaele P, Deblaere K, Achten E (2002) Motor imagery in mental rotation: an fMRI study. NeuroImage 17:1623-1633.

Wise SP, di Pellegrino G, Boussaoud D (1996) The premotor cortex and nonstandard sensorimotor mapping. Can J Physiol Pharmacol 74:469-482.

Worsley KJ, Friston KJ (1995) Analysis of fMRI time-series revisited-again. NeuroImage 2:173-181. 